\title{
RELACIÓN DE LOS EJES SUSTANTIVOS EN LA PLANIFICACIÓN ACADÉMICA EN LAS IES: CASO ECOTEC
}

\section{RELATIONSHIP OF SUBSTANTIVE AXES IN ACADEMIC PLANNING IN IHE: ECOTEC CASE}

\section{Elba Elizabeth Calderón Farfán, Mgs.} Magíster en Administración de Empresas (Ecuador). Docente Tiempo Completo de la Facultad de Ciencias Económicas y Empresariales de la Universidad Tecnológica ECOTEC, Ecuador. ecalderon@ecotec.edu.ec

\section{ARTÍCULO DE REFLEXIÓN}

Recibido: 31 de octubre de 2019.

Aceptado: 25 de noviembre de 2019.

\section{RESUMEN}

En los últimos años la evolución de la información ha dado un gran giro dentro de la educación provocando un impacto y con la necesidad de implementar nuevas estrategias de planificación. Los hechos básicos de sustentación y sostenibilidad de la calidad de la educación superior radican en las transformaciones de las matrices de organización del conocimiento de una organización académica y de los aprendizajes, produciendo que cualquier modelo de cambio fomente la integración de las funciones sustantivas de la educación superior como son formación, investigación y gestión del conocimiento formando plataformas que se enlazan en cada uno de los procesos de gestión. Al realizar un diagnóstico basado en los ejes sustantivos en la facultad de Ciencias Económicas y Empresariales de la ECOTEC se detectó una serie de deficiencias en la gestión universitaria, por lo que presenta una nueva metodología para la gestión integrada de los procesos sustantivos, la cual cuenta con seis fases y 17 etapas diseñadas con una concepción sistémica y renovada, estas reconocen las funciones del ciclo directivo, la 
dirección estratégica y concreta las actividades que se dan mediante el proyecto con su enfoque de marco lógico.

Palabras clave: ejes sustantivos, planificación estratégica, educación superior.

\section{ABSTRACT}

In recent years the evolution of information has taken a great turn in education giving an impact and seeing the need to implement new planning strategies. The basic facts of sustainability and sustainability of the quality of higher education lies in the transformation of the knowledge organization matrices of an academic organization and learning, producing that any change model fosters the integration of the substantive functions of education superior as they are training research and knowledge management forming platforms that are linked in each of the management processes. When making a diagnosis based on the substantive axes in the Faculty of Economic and Business Sciences of ECOTEC, a series of deficiencies in university management were detected, so it presents a new methodology for the integrated management of substantive processes, which counts With six phases and 17 stages designed with a systemic and renewed conception, they recognize the functions of the management cycle, the strategic direction, and concretize the activities, which are given through the project with its logical framework.

Keywords: substantive axes, strategic planning, higher education.

\section{INTRODUCCIÓN}

La sociedad actual, producto del llamado siglo de la aceleración histórica (XX), ha ido evolucionando mediante grandes transformaciones ya sea de forma positiva o negativa generadas a partir de la revolución de información y del conocimiento, a esto se podría traducir como la tercera revolución industrial. La planificación estratégica universitaria se establece basado en los tres ejes de la educación superior: formación, investigación y vinculación tomando en cuenta varias características como la integralidad, actualidad, flexibilidad, vinculación con el entorno.

ISSN: 1390-9320, Edición Especial, febrero 2020 
La planificación estratégica en las universidades tiene una gran importancia; sin embargo, a la hora de su aplicación se confrontan dificultades, que en la mayoría de los casos están dadas porque sus factores internos y los responsables de su implementación, no tienen bien definidos cuáles son sus objetivos y metas, lo que da lugar a una limitación en su puesta en marcha, en cuanto a su seguimiento y a la evaluación de sus planes.

Las universidades deben pensar en la planificación estratégica como una vía ordenada y muy bien planificada, que sea capaz de llevar a la universidad por el mejor camino, y cuando esto se logra, significa que sus directivos son actores activos de su gestión, que su planificación es constante y que el enfoque con que trabajan tiene en cuenta tanto los factores externos como internos de su gestión. De esta forma estará en condiciones de diseñar capacitaciones que erradiquen sus deficiencias; así como reflexionar con espíritu crítico de cómo se está realizando su quehacer educativo, y la percepción que de ella se tiene en el entorno en que funciona y en la comunidad de manera general. Por tanto, lo primero que debe plantearse una institución educativa cuando decide implementar un plan estratégico, es conocer a fondo cuál es su finalidad, porque con ello se pueden evitar contratiempos causados por la falta de información.

El panorama actual amerita cambios radicales en el sistema educativo, tanto en la educación primaria como la superior, dado que la necesidad de adaptar uno u otros espacios nace de cuán actualizados o desarrollados estén los espacios anteriores o paralelos. La globalización y la comunicación en masas han sido aspectos tan cruciales hoy día que las universidades a nivel mundial se han visto obligadas a adaptarse a la nueva realidad. Por esta razón, la internacionalización de la enseñanza superior es, en muchos sentidos, una respuesta al desarrollo de las nuevas tecnologías de la información y comunicación (TIC), obligando al equipo docente a adaptar sus métodos de enseñanzas a nuevas y mejor orientadas en cuanto a plataformas, dado que las generaciones contiguas necesitan estar familiarizados tanto con los contenidos como los espacios de encuentro.

ISSN: 1390-9320, Edición Especial, febrero 2020 


\section{REVISIÓN TEÓRICA}

La sociedad actual, producto del llamado siglo de la aceleración histórica (XX), ha ido evolucionando mediante grandes transformaciones ya sea de forma positiva o negativa generadas a partir de la revolución de información y del conocimiento, que se podría traducir como la tercera revolución industrial. El panorama actual amerita cambios radicales en el sistema educativo, tanto en la educación primaria como la superior, dado que la necesidad de adaptar uno u otros espacios nace de cuan actualizados o desarrollados estén los conocimientos e información en las distintas áreas educativas. La globalización y la comunicación en masas han sido aspectos tan cruciales hoy día que las universidades a nivel mundial se han visto obligadas a adaptarse a la nueva realidad.

Por ello, la internacionalización de la enseñanza superior es, en muchos sentidos, una respuesta al desarrollo de las nuevas tecnologías de la información y comunicación (TIC), obligando al equipo docente a adaptar sus métodos de enseñanzas a nuevas plataformas dado que las generaciones contiguas necesitan estar familiarizados tanto con los contenidos como los espacios de encuentro.

El estado ecuatoriano se propuso asumir como una de sus principales responsabilidades y metas, el cambio del sistema educativo en todos los niveles, por lo que se han realizado acciones puntuales caracterizadas por no disponer de un orden, ni previsión de las mismas. En los últimos años, la educación en el Ecuador está viviendo un periodo de modificaciones, sin embargo, estas han generado preocupaciones porque algunos cambios no se han realizado a tiempo y existen fallas en la calidad educativa que se han arrastrado por años, al no ajustar las medidas a las exigencias macroeconómicas de la actualidad.

La gestión académica es el conjunto de procesos aplicados en instituciones con la finalidad de alcanzar las metas propuestas por cada establecimiento en relación a la calidad educativa para lo cual se pretende trabajar a través de tres supuestos como la unidad de planteamiento estratégico que según lo menciona Donini \& De Donini (2003), suministra información cuali-cuantitativa del entorno, por su parte la gestión administrativo-financiero debería estar al servicio de desarrollo del personal no docente; así como los las prioridades expresadas en el plan estratégico institucional y la optimización de los procesos 
académicos sustantivos y, finalmente, la estructura departamental ayuda al desarrollo de las funciones académicas de enseñanza, investigación y vinculación.

Las universidades en el mundo entero se encuentran involucradas en constantes procesos de transformación, con el propósito de brindar cada vez mayores y mejores respuestas a las demandas de la sociedad; por lo que la utilización de estos conceptos y enfoques modernos de la dirección se convierten en una necesidad para desarrollar una gestión universitaria de excelencia.

Más allá de las diferencias y de las características particulares de cada una, las universidades se desarrollaron con una estructura de tipo gremial que ha marcado su impronta; pero todos se enmarcaron en una forma de organización que siempre estuvo muy definido y que subsistió durante muchos años. Según Clark (2013):

Gestionar no significa solamente adquirir el manejo de algunas técnicas. Quienes sólo hacen hincapié en las técnicas, generalmente en las de moda, para explicar la Administración de las Organizaciones sin relacionar procesos pasados con los actuales, olvidan la parte más importante de la disciplina, que consiste en situarse en el momento actual, para, con un ejercicio prospectivo, entender el sentido futuro de las decisiones presentes, con el objeto de preparar a las organizaciones para enfrentar los hechos a que se verán expuestas en el porvenir, intentando desentrañar oportunidades y amenazas que el mismo les depara (p. 153).

La gestión universitaria además de ser un concepto de un amplio espectro, debe mirarse a través de las medidas que para su efecto se establezcan. Es una teoría y una práctica científica que en el aspecto de los recursos humanos extrema sus posibilidades, asentándose en el comportamiento organizacional de la institución que sirve para evidenciar el certero servicio de la estructura que utiliza el Plan de Desarrollo Universitario, tal como lo indican Belloso y Fernandez (2013).

Quienes ejerzan la dirección ejecutiva, si quieren alcanzar buenos resultados, deberán aprender a jugar en el juego social. Pero ¿qué significa jugar bien? Esta es la pregunta 
clave para la teoría del gobierno y la planificación, porque jugar bien no solo implica el dominio intelectual de la complejidad del juego semicontrolado, sino principalmente el arte de jugar bien en la práctica, medirse con los otros jugadores y dominar la tensión que el juego produce en una situación concreta. Aquí solo se puede tratar el problema del dominio intelectual de la complejidad del juego semicontrolado. El otro aspecto aún más importante, requiere maestría artística, vocación y aptitudes que solo se prueban en la práctica política y se logran mediante el entrenamiento perseverante (p. 54).

Por otra parte, los procesos sustantivos universitarios poseen características que los diferencian de otros procesos de servicios; esto es porque son complejos y de labor adecuado, ofrecen un servicio a largo plazo a múltiples beneficiarios y deben dar respuesta a las expectativas de las partes interesadas dentro de la gestión universitaria, coexisten estructuras y esquemas de gestión diferenciados, los cuales responden a la gestión del conocimiento y son entendidos desde tres perspectivas: manejo efectivo de la información para incrementar el conocimiento, la gestión de las tecnologías e incremento del conocimiento de los profesionales que los realizan (Torres, 2010).

En la universidad se reconocen como sustantivos los procesos de formación, investigación y extensión o vinculación cuya integración permite dar respuesta plena a su misión: la docencia como proceso de enseñanza y aprendizaje de los conocimientos; producidos a través de la investigación científica representada en las diferentes ciencias y constituye el contenido de las disciplinas. La extensión universitaria interrelaciona la docencia y la investigación a través de la promoción de estas acciones al entorno social para satisfacer las necesidades del desarrollo cultural y la solución de problemas de la práctica social (Fabre, 2005).

En este sentido el modelo de gestión de la docencia es la función sustantiva que permite relacionar todos los conceptos ya descritos, bajo el amparo de la libertad de cátedra; resume las corrientes educativas; hace uso de los enfoques, las escuelas de pensamiento educativo, la intencionalidad, la perspectiva sociológica y pedagógica, la crítica como medio y la técnica como recurso.

ISSN: 1390-9320, Edición Especial, febrero 2020 
En la docencia se definen de manera funcional las operaciones básicas del proceso enseñanza-aprendizaje y se relacionan distintos elementos que confluyen hacia el fin de educar según Veras (2011), de este modo se cumplen los tres ejes sustantivos que son la formación tanto del docente como del educando, para lo cual necesita de la investigación para lograr los objetivos propuestos para su desarrollo y la extensión universitaria interrelacionada a la docencia y la investigación a través de la promoción de estas acciones al entorno social para saciar las necesidades del desarrollo cultural y la solución de problemas de la práctica social de sus conocimientos.

Los nuevos conceptos de gestión, cuentan con herramientas, técnicas e instrumentos tales como: guías, manuales, instructivos o protocolos, que unifican procedimientos, organizan la información y dejan constancia de la ejecución y resultados de gestión educativa. Una adecuada gestión educativa favorece, apoya y aprovecha el talento de los docentes brindando espacios para que organicen sus clases, actividades curriculares y evaluación de los aprendizajes, análisis y propuesta de soluciones a los problemas sociales, investigaciones relacionadas a la ciencia, actividades de vinculación con la sociedad, prácticas laborales, utilizando el tiempo adecuadamente, durante la preparación de su carrera profesional.

La planificación estratégica tiene ciertas dimensiones y lineamientos, que la institución educativa debe organizar internamente para la ejecución y planeación de los procesos académicos y de la autoevaluación institucional. En este mismo contexto, la planificación estratégica es la combinación de técnicas, instrumentos, metodologías, recursos y acciones que la institución debe aplicar para conseguir los objetivos y metas planteados. Como lo indica Caisaguano (2015):

Es importante señalar que la planificación estratégica cuenta con ciertos elementos, como por ejemplo: La ejecución de las técnicas grupales y colectivas, la comunicación interactiva y permanente con la comunidad educativa, la información permanente de los directivos para la toma de decisiones y las técnicas de autoevaluación institucional y evaluación a los estudiantes (Caisaguano, 2015). 
Para que todo esto pueda ser logrado es necesario seleccionar adecuadamente el modelo a utilizar en la planificación estratégica, y que responda a un modelo propio de la institución y evaluar la disposición y el compromiso de los directivos y docentes. La planificación estratégica ha sido utilizada por muchos años de manera universal, en la actualidad son muchos los administradores y directivos de organizaciones que la usan para corregir las actuaciones y prevenir situaciones futuras, pues esta permite realizar un pronóstico de posibles escenarios que permitirán lograr los objetivos de la planificación. En una sociedad regida por constantes cambios, es necesario dirigir las organizaciones a través de una planificación estratégica, porque esta permite:

$\checkmark$ Reconocer los cambios que se presentan en el entorno de la organización.

$\checkmark$ Aprovechar las oportunidades que ofrece el mercado.

$\checkmark$ Ajustarse a los escenarios a partir de un análisis sobre las últimas tendencias y cambios tecnológicos, políticos, sociales y económicos.

$\checkmark$ Asignar los recursos sobre una base sólida de información.

$\checkmark$ Proyectar las actividades hacia el desarrollo futuro.

$\checkmark$ Capacitar a los integrantes de la organización de forma oportuna para hacer frente a los escenarios que se van a ir presentando.

$\checkmark$ Generar cambios en el mercado y no solo adaptarse a ellos.

El proceso de planificación estratégica es muy completo, ya que abarca desde el diagnóstico de necesidades, hasta el diseño de un plan que involucra el uso de recursos financieros y las capacidades del talento humano de la organización. Logrando así la articulación de todas las potencialidades en aras del logro de los objetivos y metas de la empresa, minimizando las amenazas y pérdidas.

Como herramienta de gestión, el plan estratégico motiva a trabajar con mucha perspectiva y posicionamiento hacia el futuro, implementándolo como modelo, se regirá la principal razón de ser de la institución y hacia donde se encaminará para comenzar. Agregando los objetivos señalados y las estrategias para alcanzarlos, el mismo se debe implementar anualmente debido a que su desarrollo para ser sostenible se refleja a partir de 4-5 años.

ISSN: 1390-9320, Edición Especial, febrero 2020 


\section{MATERIALES Y MÉTODOS}

La Universidad Tecnológica Ecotec fue aprobada oficialmente el 18 de diciembre de 2006, la que desde sus inicios presentó una oferta académica distintiva y responsable con los más altos estándares de calidad educativos (ECOTEC, 2017).

La Institución cuenta con las Facultades de Derecho y Gobernabilidad, Ciencias Económicas y Empresariales, Ingenierías y Marketing y Comunicación; Posgrado y la Unidad de Educación Continua que brinda capacitaciones, cursos, talleres, etc. ya sea para diferentes beneficiarios; así como el desarrollo de propuestas de capacitación pertinentes a Vinculación con la Sociedad, permitiendo fortalecer las capacidades de la población con ambos tipos de formación (ECOTEC, 2017).

La facultad de Ciencias Económicas y Empresariales, cuenta actualmente con siete carreras: Licenciaturas en Administración de empresas, Finanzas, Comercio, Negocios Internacionales, Contabilidad y Auditoría y Gestión del Talento Humano, y la carrera de Economista.

El claustro docente está formado por 14 profesionales dedicados $100 \%$ a la docente, todos con título de 4to nivel y docente con título de Doctor en Ciencias de la Educación. Asimismo, cuenta con 7 docentes cursando estudios doctorales.

En los periodos regulares se ofertan un promedio de 140 paralelos / materias que son dictadas por docente tiempo completo como por docentes tiempo parcial (contrato bajo facturación).

Basado en los ejes sustantivos de la educación superior, se realizó un diagnóstico preliminar para determinar las insuficiencias y/o deficiencia en la gestión universitaria de la facultad de Ciencias Económicas y empresariales de la ECOTEC.

Mediante el diagrama de Causa - efecto se identificaron un conjunto de aspectos significativos que afectan la calidad de la gestión universitaria, estos son:

- Baja producción científica en la facultad lo que afecta notablemente la visibilidad de los docentes en el ámbito profesional.

ISSN: 1390-9320, Edición Especial, febrero 2020 
- Poca gestión de proyectos para trabajo con la comunidad lo que implica un bajo cumplimiento de la tercera misión de la universidad.

- No se realizan acciones de socialización del conocimiento y en particular de los productos desarrollados por los docentes.

- Inadecuado manejo de los procesos.

- Limitada estructura de supervisión y control de los procesos de gestión internos de la facultad.

- Escaso acompañamiento (consejería) al estudiante.

- No existe innovación en las metodologías aplicadas en las clases.

- Limitado aprovechamiento del talento de los docentes.

- Limitado dominio de las TIC.

- Alta rotación de docentes.

- Los organismos de control cambian constantemente los indicadores de gestión y acreditación.

En ese sentido, una gestión universitaria deficiente limita la realización de las actividades académicas, por no contar con los medios necesarios, ambientes adecuados, plataformas educativas, material de laboratorio limitado e insuficiente, simuladores (software) no actualizados, entre otros muchos elementos que provocan que no se pueda hacer un aprendizaje de calidad, tras encontrar estas deficiencias la universidad busca la manera de alcanzar la eficiencia y eficacia de sus modelos de gestión y en este contexto, es un imperativo potenciar la planificación, razón por la cual se propone implementar una metodología para mejorar la gestión integrada de los procesos sustantivos en la Facultad de Ciencias Económicas y Empresariales de la Universidad ECOTEC.

De este modo la metodología usada propuesta en esta investigación está formada por seis fases y 17 etapas las que son diseñadas con una concepción sistémica y renovada, estas reconocen las funciones del ciclo directivo y la dirección estratégica, y concreta las actividades las que se dan mediante el proyecto y su enfoque de marco lógico.

Estas fases se dan de la siguiente manera:

ISSN: 1390-9320, Edición Especial, febrero 2020 
Primera fase: inicio general del proceso, esta etapa es donde se concibe y prepara el proceso de gestión y esta se desarrolla mediante tres etapas:

1. Preparación del ambiente adecuado para el cambio

2. Preparación de las condiciones para el inicio del proceso

3. Capacitación de los actores implicados.

Segunda fase: comprende la caracterización del entorno en el que se desenvuelve y precisión de la capacidad interna del mismo, es donde se realiza el estudio del entorno y de la Facultad de Ciencias Económicas y Empresariales de la Universidad ECOTEC con el objetivo de conocer sus demandas, recursos y capacidades cognitivas y volitivas disponibles, y el ambiente para la aplicación de la DIP.

Se la formula mediante cinco etapas:

1. Precisión de la demanda interna y externa.

2. Inventario de los recursos disponibles.

3. Diagnóstico de las capacidades de los actores.

4. Diagnóstico de los órganos estructurales y funcionales.

5. Caracterización del ambiente para la DIP.

Tercera fase: se rige en la planificación, en esta se planean las acciones que deben desarrollarse para gestionar el proceso.

La planificación se realiza en cuatro etapas:

1. Definición de los escenarios de actuación.

2. Elaboración de los proyectos de gestión.

ISSN: 1390-9320, Edición Especial, febrero 2020 
3. Elaboración de los planes de acción de las estructuras de gestión y niveles de integración, y elaboración del plan de trabajo anual y mensual de la Facultad de Ciencias Económicas y Empresariales de la Universidad ECOTEC.

4. Carreras y estructuras de gestión.

Cuarta fase: esta etapa se fija en la organización, a la que se considera como la fase más importante, ya que es el lugar en donde se organiza el proceso de gestión y a la vez se desarrolla en dos etapas:

1. Diseño y creación de los órganos estructurales y funcionales para la gestión.

2. Definición de las relaciones jerárquicas entre los órganos estructurales y funcionales para la gestión.

Quinta fase: de acuerdo a esta fase es la Implementación la cual es compleja, puesto que en esta etapa se llevan a la práctica el sistema de planes concebidos y se desarrolla en una etapa la que es fundamental dentro de la misma y es la siguiente:

1. Ejecución de los planes de actividades en cada nivel, estructura y grupo.

Sexta fase: se determina la Evaluación, ajuste y mejora. A esta se la denomina como una fase determinante, pues lleva implícito un esfuerzo organizativo especial para no afectar la cohesión del equipo en torno al cumplimiento de los objetivos planificados y la que tiene como propósitos principales evaluar los resultados y realizar los cambios o ajustes necesarios.

En esta se ejecutan dos etapas las que son:

1. Evaluación de los resultados.

2. Ajuste de los objetivos y planes establecidos y propuesta de la mejora.

ISSN: 1390-9320, Edición Especial, febrero 2020 
Con esta nueva metodología se espera darle un giro al sistema para lograr los objetivos que contiene la planificación y luchar por la misión de la organización.

\section{CONCLUSIONES}

En medio del panorama formativo actual, donde se enlazan continuamente una serie de profesiones y áreas académicas, la distinción entre el mundo universitario y el laboral está cobrando importancia por cuanto se valida la verdadera experiencia en el trabajo, en lugar de que la base sea en efecto el modelo universitario. Así como la escolástica, el método educativo, ha caducado en ya muchas instituciones del mundo; la distinción maestroalumno en términos jerárquicos se está quedando atrás; de igual manera se ha ido disolviendo el viejo modo operativo de competencia del alumnado para darle sitio al modelo de grupo e inclusivo en el que se integran incluso alumnos de distintos niveles escolares con el propósito de fomentar un feedback más dinámico y nutrido que favorezca tanto en conocimientos como en perspectivas, tanto a nivel personal como relacional.

Considerando las variadas diferencias entre los modelos educativos de Ecuador y de otras regiones del mundo, y la importancia de actualizar la nacional para situar al ecuatoriano en un nivel más competitivo y apto para el mercado internacional actual. En principio, uno de los métodos más competentes tiene que ver con una reestructuración general; pero dado que eso implica un esfuerzo enorme y quizás más allá del alcance de este espacio, podría apreciarse en principio la inclusión progresiva de distintos elementos y figuras externas que confieran al sistema educativo nacional visiones y experticias de espacios más actualizados, de manera que la adaptación se dé con mayor naturalidad y se genere un encuentro de enriquecimiento mutuo, dado que la expansión de perspectivas es una de las condiciones más fértiles para la evolución de los individuos y sus sistemas.

En aras de desarrollar un más actualizado modelo educativo o, más bien, adaptar al nuevo tiempo las condiciones y metodologías de la educación superior del Ecuador, es preciso observar con detenimiento ambos sistemas, el familiar y el foráneo, con el propósito de hacer una ajustada comparativa y ver en qué se diferencian y contrastan ambos modelos entre los mejores centros del mundo. Con este propósito, la Facultad de Ciencias 
Económicas de la Universidad de Ecotec sirve como base dada la importancia que tiene para esta Casa de Estudios, pues de ella nació la institución y además cuenta con aproximadamente la mayor porción de estudiantes reunida en una sola facultad, mismo caso del profesorado; por lo cual conviene enlazar este proyecto a la misma para generar un punto de encuentro de mayor impacto, tanto para la comunidad estudiantil como para los profesionales que egresan o reconocen dicha institución.

La facultad de Ciencias Económicas y Empresariales de Ecotec debe implementar procesos que permitan mejorar la interacción de los ejes sustantivos, tratando de alcanzar la eficiencia en la gestión académica. Buscar obtener resultados eficientes, a través del cumplimiento de la planificación estratégica; así como una mejor distribución de las actividades administrativas de tal manera que los docentes puedan interrelacionar la docencia con la investigación y la vinculación.

\section{REFERENCIAS BIBLIOGRÁFICAS}

Belloso, O., \& Fernández, F. (2013). Rasfos fundamentales de los procesos de dirección en América Latina. Caracas, Venezuela: Universidad "Rafael Belloso".

Caisaguano, L. (2015). La planificación estratégica y los estándares en la gestión escolar de la Unidad Educativa Jatari Unancha. Latacunga, Ecuador: Universidad Técnica de Cotopaxi.

Clark, B. (2013). El sistema de Educación Superior. México: Nueva Imagen.

Donini, A., \& A M De Donini. (2003). La gestión universitaria en el siglo XXI. 15. Recuperado de http://repositorio.ub.edu.ar/bitstream/handle/123456789/393/107_donini.pdf?seque nce $=2 \&$ is Allowed $=y$

ECOTEC. (marzo de 2017). Plan Estratégico de Desarrollo Institucional (PEDI). Recuperado de https://www.ecotec.edu.ec/pedi/

Fabre. (2005). Las funciones sustantivas de la universidad y su articulación en un departamento docente. In V Congreso Internacional Virtual de Educación. En U.

ISSN: 1390-9320, Edición Especial, febrero 2020 


\begin{abstract}
A. Habana. Cuba. Recuperado de
http://vitela.javerianacali.edu.co/bitstream/handle/11522/11327//ntegracion_funcion es_sustantivas.pdf?sequence $=2 \&$ isAllowed $=y$
\end{abstract}

Torres, C. E. (2010). Retos del postgrado en las universidades públicas. En U. A.

Habana, Gestión de los procesos sustantivos en la Universidad Cubana actual (p.

22). México. Recuperado de https://www.gestiopolis.com/gestion-de-los-procesossustantivos-en-la-universidad-cubana-actual/

Veras, H. A. (2011). Modelo Educativo de la UAEH. En U. A. Hidalgo. Recuperado de https://www.uaeh.edu.mx/docencia/docs/modelo_educativo_UAEH.pdf 